\title{
Emotional Memory in Alzheimer's Disease and Its Atypical Neural Mechanism
}

\author{
Letian $\mathrm{Zhu}^{1, *}$ \\ ${ }^{1}$ Xi'an Gao Xin No.1 High School, Shaanxi, China \\ ${ }^{*}$ Corresponding author. Email: oliviazhu@st.btbu.edu.cn
}

\begin{abstract}
Emotions support the formation of durable representations of past experiences in long term memory. It has been suggested that people tend to have a better memory performance for emotional stimuli than neutral stimuli. Based on previous studies, the uncinate fasciculus, linking the amygdala and temporal lobe with the prefrontal lobe, is critical for emotional episodic memory. Alzheimer' s disease is a neurodegenerative disease that will interfere with patients' cognitive function, such as deterioration in memory. However, limited research has prioritised AD patient' s emotional memory performance. In this case, this review aims to better understand the impact of AD on emotional memory from both behavioural and neural mechanism levels. Researchers wondered whether emotional memory deficit would be found in $\mathrm{AD}$ patients; whether the memory enhancement effect of emotion is also reduced in AD patients; and weather it could be enhanced intentionally by deep encoding. Additionally, this review summarized the abnormal neural mechanisms. Previous researchers have investigated the damage of white matter connections and grey matter of the limbic system networks associated with AD patients' emotional memory deficits. Moreover, researchers suggest that, for the emotional stimuli, AD patient's initial encoding process is different from healthy controls. Last but not least, the investigation has been focused on the effect of neurofeedback whether it could enhance the emotional memory of AD patients as that of healthy subjects. Nevertheless, intactness is required for a successful application of neurofeedback. Thus, such implementations should be applied as early as possible in the course of the disease to ensure optimal results. The future direction should emphasize more causal researches, considering the real social context and tracing and assessing subsequent emotional memory performance after the intervention.
\end{abstract}

Keywords: Emotional memory, Alzheimer's disease, Abnormal neural mechanism, Neurofeedback intervention

\section{INTRODUCTION}

Emotional memory is a memory for events that evoke a response with emotional valance [1]. Memories about emotional stimuli are often stored in both kinds of systems. Emotion events can trigger out the most vivid autobiographical memories and have a powerful effect on humans and animals. A phenomenon well known as an emotional enhancement of memory (EEM) describes those emotional stimuli that are more likely to be remembered than neutral stimuli [1]. Emotion is thought to support the formation of stable and durable representations of past experiences in long term memory [2]. Survival depended on learning from past experiences so that survival and evolution were both in favour of the value of emotions. For most of the time, emotional memory is not explicitly recognized, but it plays an exceedingly important role in people' $\mathrm{s}$ decision- making, learning and other memories. The emotions can be roughly divided into two categories: positive and negative. When the human brain is in a positive emotional condition, more neuronal networks are activated, nerve conduction velocity will be upgraded, and the whole brain will reach a very active and exciting state. The brain will record these "feelings", thus forming a deep "emotional memory". The active and excited state of the brain makes learners focus on their attention, enhance their learning motivation, have lively and diverse thinking activities, and have a positive attitude to solve problems. The working efficiency will increase exponentially. With regard to the neural mechanisms, neural structures that govern emotional memory involve extensive distributed connections between the amygdala and other medial temporal lobe structures, as well as the prefrontal and sensory regions, which work with the 
norepinephrine, dopaminergic, and glucocorticoid neuroregulatory systems to enhance or impair memory [3]. Based on the previous study, the uncinate fasciculus links the amygdala and temporal lobe with the prefrontal lobe, which is critical for episodic memory [4]. Alzheimer's Disease (AD) is a neurodegenerative disease that usually starts slowly and progressively worsens [5]. Emotional memory is essential to $\mathrm{AD}$ patients since it relates to the ability of processing and retrieving emotional information, which plays a role in patients' daily interactions with others.

It is the most common type of dementia in the senile, characterized by difficulty in remembering recent events in an early stage and subsequent progressive impairment in cognitive ability, such as speech degradation, disorientation, loss of motivation, self-neglect, and other behavioral issues, as the disease advanced [5, 6]. The precise causes of AD still remain unclear, and there have been several competing theories. The amyloid cascade model serves as the backbone of the current understanding of the pathogenesis of $\mathrm{AD}$. It suggests that the accumulation of $A$-protein is the core leading factor of observed neurodegeneration. Another neuropathological hallmark of $\mathrm{AD}$ is neurofibrillary tangles mainly composed of abnormally phosphorylated tau protein, a normal axonal protein that has an essential role in microtubules' assembly and stability. The abnormal accumulations and tangles of proteins result in the death of neurons under certain regions of the cortex, which is represented as brain atrophy. Moreover, three genes have been recently known to be relevant to the inherited forms of $\mathrm{AD}$. Mutations in the gene encoding for the amyloid precursor protein (APP) were the first to be identified in 1991 [7, 8]. Nevertheless, most inherited cases were caused by mutations in the genes encoding for presenilin-1 and presenilin-2 (PSEN1 and PSEN2), parts of the $\gamma$-secretase complex [8, 9]. Besides, the gene encoding for apolipoprotein E (APOE) was known to play a role in the pathogenesis of sporadic AD [10]. AD is an extremely frequently debated topic, as well as a vital issue to pay efforts on. According to the statistics conducted by WHO, AD and other dementias were ranked at the fifth place of the Top 10 Global cause of Deaths, 2016 [11].

Since AD is an irreversible disease and has no clear medical solutions yet, once the older get this disease, the cognition, speech, memory, and self-awareness will be all on the decline. There will be a great mental and economic burden on their families. As the ageing process continues to be serious, Alzheimer's disease, a thorny social problem, will be more prevalent shortly. As the existing study suggested, emotion plays a positive role in people' s memory performance, which means people are more aware of events or feelings with emotional valance. It was observed that the enhancing effect of emotion on memory tends to be preserved among older healthy adults, and the amygdala shows relatively less degeneration than many other brain regions [12]. In that way, if emotional memory deterioration has been observed in AD patients, normal ageing may not serve as a possible and reasonable explanation, and the negative impact of cognitive pathology or dementia could be revealed. However, previous studies mainly focused on the effect of AD on semantic memory, episodic memory, and implicit memory. Progressive collapse of semantic memory becomes apparent in mild $\mathrm{AD}$, presenting as difficulties in confrontation naming and in generating verbal definitions; progressive deterioration of episodic memory is typically found in $\mathrm{AD}$ from the incipient phase [13]. On the contrary, portions of implicit memory are relatively maintained even in severe $A D$ [14]. In comparison, relatively limited research has been focused on AD patient' s emotional memory performance and how AD affects the neural structures related to patient' $\mathrm{s}$ emotional memory. In this case, this review aims to better understand the impact of $\mathrm{AD}$ on emotional memory at both behavioral and neural mechanism levels.

\section{EMOTIONAL MEMORY DEFICIT IN ALZHEIMER'S DISEASE}

As previous studies suggested, semantic and episodic memories are both found to deteriorate in AD patients. It is predicted that Alzheimer's disease may also impair patients' emotional memory. In the study by Melendez et al., the influence of certain cognitive pathologies, such as mild cognitive impairment (MCI) and $\mathrm{AD}$, on emotional components of autobiographical memory (AM) was assessed [15]. Experimenters used an autobiographical memory test to measure the difference in responses to emotional cues. The recruited samples were divided into three groups: healthy older adults (HOA) matching on other required conditions, adults with $\mathrm{MCI}$, and adults with moderate AD. Participants were given two lists of words with opposite valance, positive and negative. An interview followed the encoding of words, and participants were required to respond to each word, describing what matters had happened in their days relevant to the word given. The result revealed significant differences, among groups, in the retrieval of memory with different valance. HOA and MCI recalled significantly more events based on given emotional words than samples with $\mathrm{AD}$ did. In short, it can be concluded that AD patients have a decline in emotional memories.

With regard to the deterioration of emotional memory in $\mathrm{AD}$ patients, researchers were investigating whether the memory enhancement effect of emotion is also reduced. Chainay et al. experimented to evaluate EEM (an effect mentioned above that emotional stimuli could trigger enhanced memory performances) in $\mathrm{AD}$ while manipulating intention [16]. The stimuli were color photographs of common man-made and natural elements. 
In the experiment, the intention was manipulated by informing participants about the retrieval process following the categorization task. In the categorical task, participants need to choose whether the element belongs to the natural or man-made semantic category. After encoding, participants were asked to do an immediate recognition task, quickly judging whether they had seen the picture presented in previous encoding tasks. It is found that, in both intentional and incident conditions, the AD group showed significantly worse emotional memory than the control group. In both conditions, AD patients did not present significant differences in response to photographs with different valence. On the contrary, the control group showed better memory for positive stimuli than neutral and negative stimuli, especially in the intentional condition. The result suggested that $\mathrm{AD}$ patients didn't present a distinct emotional enhancement effect so that they don't have better memory performance for emotional stimuli.

Although the emotional enhancement effect wasn't naturally observed in $\mathrm{AD}$ patients, researchers wondered whether the effect could be enhanced through training. The study conducted by Sava et al. was designed to ascertain whether rich and deep encoding might permit the emergence of EEM in AD [17]. Mild-to-moderate AD patients and controls matching age and education participated in two encoding phases and the following two retrieval phases. The stimuli were pictures selected from a pool of photographs with emotional valence and arousal. During the first study phase, participants performed a natural/man-made categorization task. Then, a recognition task followed. During the second study phase, participants were asked to name the object in the picture, which had shown up in the first retrieval, and to read aloud the object's category. The second encoding phase was followed by a free recall task, a cued recall task during which the semantic encoding in the second study phase is the cue, and a recognition task. In both the first recognition and free recall tasks, the control samples were significantly better at recognizing positive and negative stimuli than neutral ones. By contrast, AD patients show no difference to different valence in both tasks. Interestingly, after deeper encoding, the positivity memory bias was more striking in $\mathrm{AD}$ patients than in controls in the cued-recall task and the following recognition task. $\mathrm{AD}$ patients remembered positive stimuli better than both negative and neutral stimuli. At the same time, the control group showed a significant advantage in recognising positive pictures over negative pictures, but not over neutral ones. The present study is the first to demonstrate that the emergence of the positivity memory bias is possible in mild $\mathrm{AD}$ patients when deep encoding conditions are permitted and when support is provided at the retrieval processes. Consequently, although EEM is found to be impaired in $\mathrm{AD}$ patients, intentional, deep encoding and intervention could help improve AD patients' emotional memory.

\section{ABNORMAL NEURAL MECHANISM AND THE RELEVANT INTERVENTION}

Memory and emotion are closely related to the functions of the Medial Temporal Lobe (MTL), especially the amygdala and hippocampus [18]. The hippocampus serves a crucial role in short-term memory and consolidates that memory into a long-term form. The amygdala is a collection of nuclei in the temporal lobe. Considerable evidence from both human and experimental animals now indicates that, concerning conscious feelings, especially fear, the amygdala engages with the cortical areas, involving cingulate, parahippocampal, and prefrontal cortices. The existing studies have found the difference in emotional memory, in behavioral level among $\mathrm{AD}$ patients and healthy controls. Thus, it is important to investigate whether differences between $\mathrm{AD}$ patients and controls also exist in related neural structures, like the amygdala. Nathalie and her colleagues studied the influence of emotions on autobiographical memory $(\mathrm{AbM})$ in patients with $\mathrm{AD}$, characteristically triggering atrophy in the hippocampus and the amygdala [18]. The study measured the emotional $\mathrm{AbM}$ and amygdala-hippocampal volumes using the modified Crovitz test (MCT) and magnetic resonance imaging (MRI). Participants consisted of early-to-mild $\mathrm{AD}$ patients and age-matched healthy controls. The researchers obtained 30 autobiographical memories per participant by asking participants to produce detailed recollections in response to 6 specific cues, each used five times to elicit memories from five life periods. During the recollection, amygdalahippocampal volumes of AD patients were extracted and used to determine correlations with emotional scores. It is found that there is a positive correlation between emotional AbM loss in AD patients and the degree of atrophy of the right amygdala and hippocampus. Therefore, the atrophy could be found in AD patients' brain structures related to emotional memory processing

The above study showed the atrophy in related neural structures in $\mathrm{AD}$ patients. Researchers wondered whether $\mathrm{AD}$ and MCI patients show the difference in the encoding process of emotional contents with the healthy controls. Parra et al. chose a task previously reported by Whalley et al., which investigates emotional memory for positive and neutral scenes using an incidental memory paradigm [19]. Patients with MCI, AD and Healthy Controls (HC) were asked to attend to emotional pictures (i.e., positive and neutral) sequentially presented during an fMRI session to measure their brain activation during incidental encoding. The scanning session was divided into blocks, including images of positive emotional scenes, images of a neutral emotional scene, and baseline scenes. A surprising recognition task was conducted immediately after the scan. After analyzing the scanning neuroimages and behavioral performance in the recognition task within and between groups, researchers found that $\mathrm{HC}$ 
was the only group showing significantly better incidental memory in emotional scenes than in neutral scenes. Via a within-group analysis with all scenes versus baseline, researchers have found significant activation clusters resulting from the emotional memory paradigm in MTL structures and medial frontal regions, in HC and MCI, but not in AD. Via a between-group analysis, with the contrast of all scenes versus baseline, the AD patients demonstrated declined activation of the PHG/Hippocampal region versus both the control and MCI groups. As Parra's behavioral measures showed, the most impaired pattern of functional activation was found in the $\mathrm{AD}$ group, and they showed no behavioral benefit stemming from the emotional stimuli. A potential account for this functional impairment could be a poor encoding of emotional information. In other words, it might be possible that $\mathrm{AD}$ and MCI patients perceive the emotional component of the pictures differently or insufficiently as HC did. In that case, Parra and his colleagues tested this hypothesis in a subgroup of participants (5 HC, $7 \mathrm{MCI}$ and $3 \mathrm{AD}$ patients) who were asked to assess the images after the post-scan test as emotional or non-emotional [19]. As a result suggested, $\mathrm{AD}$ patients showed a significant difference in the rating performance between emotional and neutral categories. MCI also had such a pattern, but the difference is not as significant as that presented by AD. By contrast, the amounts of emotional stimuli and neutral stimuli rated by $\mathrm{HC}$ were virtually approximate. Based on the result, it is possible to interpret that $\mathrm{AD}$ and $\mathrm{MCI}$, in fact, show impairment even during the encoding and processing process of emotional information. In other words, the conclusion could be reached that the atypicality in stimuli processing influences further memory performance of $\mathrm{AD}$ patients.

In the further investigation, $\mathrm{Li}$ et al. were devoted to observing whether EEM is absent in amnestic MCI (aMCI) and $\mathrm{AD}$ patients and to investigate if emotional memory is associated with white matter (WM) connections and grey matters (GM) of the limbic system networks [20]. AD patients, aMCI patients, and normal controls participated in the Emotional Memory Behavior test involving encoding, retrieval, and MRI scanning. The encoding process involved a categorization task containing stimuli (pictures) varying in emotional valance. Participants were asked to determine the number of people in the photographs immediately. An incident recognition test followed the categorization task. Based on the emotional memory behavior test results, both previously seen positive and negative stimuli were discriminated by NC participants; aMCI patients solely discriminated against positive stimuli; while AD patients showed no discrimination over any valance of stimuli. Based on fMRI neuroimages, researchers observed the pattern of GM and WM in three groups of participants. The impairments in the limbic system networks were found in $\mathrm{AD}$ patients. Compared with $\mathrm{NCs}, \mathrm{AD}$ patients showed a widespread pattern of GM atrophy, while the impairments of aMCI patients were more localized and milder. Besides, the results confirmed that the integrity of uncinate fasciculus was also associated with the discrimination of previous seen emotional pictures in $\mathrm{AD}$ patients. By the analysis of correlations, significant correlations between the bilateral uncinate fasciculus, corpus callosum (genu and body), left cingulum bundle, left parahippocampal WM and the recognition sensitivity of emotional valence pictures; and significant correlations between the splenium of the corpus callosum, left cingulum bundle, left crus of fornix and stria terminals and the recognition sensitivity of EEM were found. Therefore, it can be confirmed that abnormality in the GM and WM connections of those structures mentioned above is associated with impaired emotional memory and EEM effect in AD patients.

As suggested by a previous study, cognitive training can, to some extent, reverse cognitive decline. Thus, recently, in the context of real-time functional magnetic resonance imaging (rtfMRI), neurofeedback has been established. Neurofeedback is an intervention method in which brain activation states and the discrepancy from the reference activation of normal people are visualized to subjects while they are performing the task. After being informed, subjects will try to modulate or train their brain activation to approach to the ideal extent. The validity of neurofeedback is widely accepted since it has been practised in many clinical and non-clinical applications. For instance, previous studies on healthy subjects announced improved working memory performance after rtfMRI neurofeedback training of the dorsolateral prefrontal cortex [21, 22]. The healthy elderly and patients with prodromal AD showed improved visuospatial memory performance after neurofeedback training. However, limited researches have investigated neurofeedback intervening in the emotional memory performance. In that case, Researchers wondered whether neurofeedback would also enhance the effect on the emotional memory of both $\mathrm{AD}$ patients and healthy subjects.

Hohenfeld and his colleague designed a study applying rtfMRI neurofeedback training targeting the left PHG and visuospatial memory in the healthy elder group and the $\mathrm{AD}$ group to improve cognitive performance. Their protocol consists of five examination days (T1-T5): a pre-test at $\mathrm{T} 1$, intervention at $\mathrm{T} 2-\mathrm{T} 4$, and a post test at T5. At T1, a visuo-spatial memory task of encoding a real-world footpath and an anatomical MRI scan of the brain was performed. The instruction was to memorize as many path details as possible while being guided by experimenters along the path. For every 10 waypoints, there is a stop (prominent landmarks, usual buildings with unique features). At each stop, subjects were informed about the waypoint's name, and they were required to specifically memorize the waypoint. As soon as subjects confirmed that they had well memorized the 
waypoint, the tour continued. In the intervention process, an ROI within the left PHG was selected as the training target for groups $\mathrm{HC}$ and PA. A region from the left primary somatosensory cortex was chosen as the target for group SH. Brain activation derived from the selected ROI was visualized as a thermometer bar during neurofeedback training runs. Upregulation phases required subjects to actively remember and imagine the path they had learned at T1 to enhance brain activation visualized in the thermometer bar. Baseline phases required subjects to slowly count backwards from 100 to bring down the thermometer bar. Finally, T5 involved a neuropsychological examination and another anatomical scan of the brain. It is reported that the healthy elderly and patients with prodromal $\mathrm{AD}$ showed improved visuospatial memory performance after neurofeedback training. In $\mathrm{HC}$ and PA groups, activation of the target region left PHG was revealed, but not in $\mathrm{SH}$. In $\mathrm{HC}$, activation was further found in the right precuneus, left posterior cingulate, right PHG, bilaterally in the superior occipital gyrus and middle frontal gyrus, as well as in cerebellar areas. In summary, this study implies that by the intervention of rtfMRI neurofeedback training paradigms, subjects, both AD patients and the healthy old, are possible to improve their emotional memory performance by activating related brain areas. Therefore, this approach of neurofeedback training could be used to counteract the cognitive effects of both $\mathrm{AD}$ and healthy ageing.

\section{LIMITATION AND FUTURE DIRECTION}

Several limitations of the existing studies discussed above were revealed. Firstly, most of the previous researches are correlational studies, so the study of a more direct and closer relation between $\mathrm{AD}$ and emotional memory is insufficient. Future studies should focus more on the cause and effect. The second limitation is the testing paradigm. Most of the emotional measurements have been done by lab testing, asking subjects to look at cue pictures and recall previous related events, which contains a gap from reality. In future studies, more tasks should be done under social scenarios to enable subjects to perform as normal as possible, thus generating the most accurate results. Another limitation is that previous researches had mainly focused on crosssectional studies, which focused on a certain section of a patient's developmental stage. Limited follow-up assessment has been done to test whether these observed effects, like enhancing memory after fMRI-based neurofeedback training, would persist in the long run. It is unclear that whether the role of emotional memory varies during different stages of $\mathrm{AD}$. The development of emotional memory deficit remains ill-defined as well, whether it continues all along with the development of $\mathrm{AD}$ or doesn't show up in the early stage. Moreover, it is also worth investigating whether there is a correlation between emotional memory and other types of memories; if any, whether this correlation would change with $\mathrm{AD}$ development. Therefore, future studies should be supplemented with follow-up and longitudinal investigations, measuring the developmental stages of emotional memory deficit in $\mathrm{AD}$ patients to verify the subsequent influence of intervention.

\section{CONCLUSION}

It can be concluded that $\mathrm{AD}$ patients have impaired emotional memory and EEM effect, so different from the healthy old, they don't present better memory performance for emotional stimuli. Nevertheless, it is observed that intentional, deep encodings and intervening trainings could help improve AD patients' emotional memory. It is found that the atypicality in stimuli processing also affects further emotional memory performance. Regarding neural structures, the atrophy could be found in $\mathrm{AD}$ patients' brain structures related to the storage and processing of emotional memory. Deteriorated emotional memory and EEM effect of AD patients could be explained by damages found in the GM and WM connections of limbic system networks, uncinate fasciculus, corpus callosum (genu and body), left cingulum bundle and left parahippocampal. Furthermore, with the use of the intervention of rtfMRI neurofeedback training paradigms, subjects, both AD patients and the healthy old, are possible to improve their emotional memory performance by practicing activating targeted brain areas. This approach could be applied to counteract the cognitive effects of $\mathrm{AD}$ as early as possible in the course of the disease to ensure optimal results. Regarding the limitations from three aspects, including being restrained in correlation study, using lab testing, and without subsequent tracing over the effect of the intervention, future studies should contain more investigations focusing on cause-and-effect relation, conducting in social scenarios, and following up longitudinal development.

\section{REFERENCES}

[1] Hamann, S. (2001). Cognitive and neural mechanisms of emotional memory. Trends in cognitive sciences, 5(9), 394-400.

[2] Dere, E., Pause, B. M., \& Pietrowsky, R. (2010). Emotion and episodic memory in neuropsychiatric disorders. Behavioural brain research, 215(2), 162171.

[3] Taoka, T., Morikawa, M., Akashi, T., Miyasaka, T., Nakagawa, H., Kiuchi, K., ... \& Kichikawa, K. (2009). Fractional anisotropy - threshold dependence in tract-based diffusion tensor analysis: evaluation of the uncinate fasciculus in Alzheimer 
disease. American

Journal

of

Neuroradiology, 30(9), 1700-1703.

[4] Fujie, S., Namiki, C., Nishi, H., Yamada, M., Miyata, J., Sakata, D., ... \& Murai, T. (2008). The role of the uncinate fasciculus in memory and emotional recognition in amnestic mild cognitive impairment. Dementia and geriatric cognitive disorders, 26(5), 432-439.

[5]Mucke, L. (2009). Alzheimer's disease. Nature, 461(7266), 895-897.

[6] Burns, A., \& Iliffe, S. (2009). Alzheimer' s disease. BMJ 338, b158.

[7] Goate, A., Chartier-Harlin, M. C., Mullan, M., Brown, J., Crawford, F., Fidani, L., ... \& Hardy, J. (1991). Segregation of a missense mutation in the amyloid precursor protein gene with familial Alzheimer's disease. Nature, 349(6311), 704-706.

[8] Papassotiropoulos, A., Fountoulakis, M., Dunckley, T., Stephan, D. A., \& Reiman, E. M. (2006). Genetics, transcriptomics, and proteomics of Alzheimer's disease. Journal of Clinical Psychiatry, 67(4), 652.

[9] Serretti, A., Olgiati, P., \& De Ronchi, D. (2007). Genetics of Alzheimer's disease. A rapidly evolving field. Journal of Alzheimer's Disease, 12(1), 73-92.

[10] Irizarry, M. C., Deng, A., Lleo, A., Berezovska, O., Von Arnim, C. A., Martin - Rehrmann, M., ... \& Rebeck, G. W. (2004). Apolipoprotein E modulates $\gamma$ - secretase cleavage of the amyloid precursor protein. Journal of neurochemistry, 90(5), 11321143.

[11] World Health Organization. (2018). Global Health Estimates 2016: Deaths by cause, age, sex, by country and by region, 2000 - 2016. Geneva: World Health Organization.

[12] Mather, M. (2004). Aging and emotional memory. In D. Reisberg and P. Hertel, (Eds.) Memory and Emotion. NY: Oxford University Press, 272-307. Small, B. J., Mobly, J. L., Laukka, E. J., Jones, S., \& Bäckman, L. (2003). Cognitive deficits in preclinical Alzheimer's disease. Acta Neurologica Scandinavica, 107, 29-33.

[13] Hodges, J. R., Patterson, K., Graham, N., \& Dawson, K. (1996). Naming and knowing in dementia of Alzheimer's type. Brain and language, 54(2), 302325 .

[14] Faul, L., \& LaBar, K. S. Emotional Memory in the Human Brain. In The Oxford Handbook of the Neurobiology of Learning and Memory.
[15] Meléndez, J. C., Escudero, J., Satorres, E., \& Pitarque, A. (2019). Type of memory and emotional valence in healthy aging, mild cognitive impairment, and Alzheimer , s disease. Psicothema, 31(1), 60-5.

[16] Chainay, H., Sava, A., Michael, G. A., Landre, L., Versace, R., \& Krolak-Salmon, P. (2014). Impaired emotional memory enhancement on recognition of pictorial stimuli in Alzheimer's disease: no influence of the nature of encoding. cortex, 50, 32-44.

[17] Sava, A. A., Paquet, C., Krolak-Salmon, P., Dumurgier, J., Hugon, J., \& Chainay, H. (2015). Emotional memory enhancement in respect of positive visual stimuli in Alzheimer's disease emerges after rich and deep encoding. cortex, 65, 89-101.

[18] Philippi, N., Botzung, A., Noblet, V., Rousseau, F., Després, O., Cretin, B., ... \& Manning, L. (2015). Impaired emotional autobiographical memory associated with right amygdalar-hippocampal atrophy in Alzheimer' s disease patients. Frontiers in aging neuroscience, 7, 21.

[19] Parra, M. A., Pattan, V., Wong, D., Beaglehole, A., Lonie, J., Wan, H. I., ... \& Lawrie, S. M. (2013). Medial temporal lobe function during emotional memory in early Alzheimer' $s$ disease, mild cognitive impairment and healthy ageing: an fMRI study. BMC psychiatry, 13(1), 1-12.

[20] Li, X., Wang, H., Tian, Y., Zhou, S., Li, X., Wang, K., \& Yu, Y. (2016). Impaired white matter connections of the limbic system networks associated with impaired emotional memory in Alzheimer's disease. Frontiers in aging neuroscience, 8, 250.

[21] Zhang, G., Yao, L., Zhang, H., Long, Z., \& Zhao, X. (2013). Improved working memory performance through self-regulation of dorsal lateral prefrontal cortex activation using real-time fMRI. PloS one, $8(8)$, e73735.

[22] Sherwood, M. S., Kane, J. H., Weisend, M. P., \& Parker, J. G. (2016). Enhanced control of dorsolateral prefrontal cortex neurophysiology with real-time functional magnetic resonance imaging (rt-fMRI) neurofeedback training and working memory practice. Neuroimage, 124, 214-223. 